\title{
Communication brève
}

\section{Evaluation préliminaire des besoins de formation en gastroentérologie des médecins urgentistes au sein d'un centre hospitalo-universitaire}

\author{
Preliminary Evaluation of Continuing Medical Education Needs in Gastroenterology \\ Emergency Practice Within a University Hospital Environment
}

\author{
Véronique VITTON ${ }^{1}$, Pascal VIGNALLY², Stéphanie GENTILE², Maéva DELAVEAU',
} Jérôme POUSSARD', Marc BARTHET

\begin{abstract}
Résumé But : Depuis plusieurs années en France, s’est développée une offre de formation médicale continue destinée au perfectionnement des médecins libéraux. En revanche, au sein des Centres hospitalo-universitaires, il néexiste pas souvent de dispositifformel de formation spécifiquement destiné aux médecins généralistes urgentistes. Le but de notre travail était d'évaluer les besoins de formation en hépato-gastro-entérologie des médecins urgentistes de notre centre. Méthodes : Notre démarche d'évaluation des besoins sest appuyée sur des entrevues individuelles et un questionnaire ouvert complété par une grille d'analyse quantitative et qualitative (grille Fréquence, Gravité, Problème). L'avis des gastroentérologues a également été recueilli grâce aux mêmes questionnaires. Résultats : Les réponses de 22 médecins urgentistes et des six gastroentérologues ont pu être recueillis. Les entrevues individuelles ont permis de mettre en évidence des besoins de formation ressentis par l'ensemble des médecins urgentistes. La réunion préalable à la remise des questionnaires a permis d'individualiser trois sujets (hémorragie digestive, pancréatite aiguë, angiocholite aiguë). Pour l'ensemble des sujets considérés, les médecins urgentistes exprimaient des besoins en termes de savoir et de savoir-faire mais pas de besoin dans le registre du savoir-être. En revanche, les gastroentérologues exprimaient, pour les médecins urgentistes, des besoins concernant les trois dimensions pour chaque problème. Conclusion : Notre travail a permis de mettre en évidence des besoins ressentis et exprimés de formation de la part des médecins urgentistes. L'utilisation ultérieure d'outils complémentaires d'analyse des besoins devrait nous permettre de préciser encore mieux ces besoins afin de développer une formation adéquate.
\end{abstract}

Mots clés Formation médicale continue ; évaluation des besoins de formation ; gastroentérologie ; service d'urgences.

Abstract Aim: For several years, continuing medical education has been developed in France to support private practice physicians' improvement. However, within a university hospital centre environment, continuing medical education does not exist, specifically towards emergency physicians. The aim of our study was to evaluate educational needs for gastroenterology practitioners working in the emergency room. Methods: Evaluation of continuing medical educational needs was obtained from individual interviews and an open questionnaire completed with a qualitative and quantitative grid analysis. Advices from gastroenterology practitioners were also collected from the same questionnaire. Results: Opinions from 22 emergency physicians and from 6 gastroenterology practitioners were collected. Answers from individual interviews including all emergency physicians brought to light needs for continuing medical education. A previous briefing meeting before questionnaire distribution allowed us to identify three subjects for continuing medical education: digestive haemorrhage, acute pancreatitis and acute angiocholitis. For all of those subjects, emergency practitioners expressed educational needs concerning knowledge and knowing-how but they did not point out needs relating to knowledge being. However, for each subjects, gastroenterologists expressed continuing medical education needs for all dimensions of knowledge. Conclusion: Our work allowed us to raise continuing medical educational needs, which was felt and expressed by emergency physicians. The use of future analysis systems to evaluate further needs, will help us to better identify these needs in order to develop an appropriate continuing medical education.

Keywords Continuing medical education; evaluation of continuing medical education needs; gastroenterology; emergency room.

Pédagogie Médicale 2007;8:107-14 


\section{Communication brève}

\section{Introduction}

L'évolution régulière de la médecine nécessite une actualisation permanente des connaissances et des compétences de la part des praticiens. En France, cette notion est stipulée dans l'article $11 \mathrm{du}$ code de déontologie (article R.4127-11 du code de la santé publique), invitant le praticien à " prendre toutes dispositions nécessaires pour participer à des actions de formation continue et à participer à l'évaluation des pratiques professionnelles ${ }^{1}$. Depuis ces dernières années, cette exigence a été renforcée par des dispositions législatives ayant pour but de structurer et d'organiser de manière méthodique cette obligation du praticien, en prescrivant un double engagement dans des activités de formation médicale continue (FMC) et d'évaluation des pratiques professionnelles (EPP) ${ }^{2}$.

Néanmoins, au sein de la plupart des centres hospitalouniversitaires (CHU), où cohabitent de nombreux services de spécialités médicales et chirurgicales, il n'existe pas dispositif formel de formation développée par les services spécialisés à l'intention des médecins généralistes qui travaillent dans le service d'accueil des urgences. En effet, les connaissances médicales nécessaires dans le cadre de la médecine d'urgence au sein d'un CHU ne font pas encore, à ce jour, l'objet d'un enseignement postuniversitaire structuré puisque la spécialité médicale "d'urgences " n'a été que récemment définie. Nous avons pu observer, au sein de notre CHU, une discordance non négligeable entre la prise en charge des patients aux urgences et celle préconisée par les services de spécialités, en particulier dans le domaine de la gastroentérologie. La mise en œuvre d'une formation en gastroentérologie destinée aux médecins urgentistes paraît donc nécessaire pour optimiser la prise en charge médicale initiale de patients souffrant de pathologies digestives et admis dans le service des urgences. Plusieurs étapes sont nécessaires au développement d'un projet de formation. Il faut, en effet, identifier les besoins de formation puis déterminer les objectifs d'enseignement, le choix des apprenants, le choix des méthodes pédagogiques et des méthodes d'évaluation.

Le but de notre travail est de rapporter une démarche exploratoire visant à recenser des besoins de formation en hépato-gastro-entérologie adaptés à des médecins urgentistes travaillant au sein de notre $\mathrm{CHU}$, en faisant référence aux compétences professionnelles visées ${ }^{3-5}$.

\section{Méthodes}

Notre étude s'inscrit dans le cadre d'une démarche exploratoire pilote, au sein d'un service d'urgence au sein du CHU Nord de Marseille.

\section{Contexte}

Le service des urgences de l'hôpital Nord est constitué de quinze médecins généralistes titulaires du diplôme d'état de docteur en médecine (médecins dits "seniors "), de dix internes de médecine générale et de dix étudiants hospitaliers de deuxième année du deuxième cycle des études médicales (DCEM2). Lors de chaque garde sont présents deux médecins seniors, trois internes et quatre étudiants. Nous avions exclu de notre travail les étudiants hospitaliers dont la formation est dépendante du service dans lequel ils sont affectés, leur apprentissage répondant à des objectifs déterminés au niveau national par le ministère au sein d'un apprentissage mixte (à l'hôpital et à la faculté) ${ }^{6}$. Cette formation a d'ailleurs bien été développée et complétée par l'équipe médicale des urgences de l'hôpital de la Conception de Marseille ${ }^{7}$. En moyenne, 130 patients sont admis quotidiennement dans le service des urgences de l'hôpital Nord. Les pathologies digestives y sont fréquentes et posent souvent un problème diagnostique et thérapeutique pour les médecins urgentistes. Les moyens de communication actuels entre médecins urgentistes et gastroentérologues sont de deux types.

Le premier moyen est représenté par les avis spécialisés dispensés à la demande des médecins urgentistes dans la journée. La nuit, seule une astreinte d'endoscopie est prévue. Le deuxième moyen de communication est un manuel disponible dans le service des urgences, contenant des protocoles de conduites médicales et chirurgicales à tenir, rédigés par les différents services spécialisés de l'hôpital. Néanmoins, ce manuel est peu réactualisé ; de plus, le niveau variable de formation des urgentistes

1- Service d'hépato-gastroentérologie, Hôpital Nord, Marseille

2- Laboratoire de Santé Publique, Faculté de Médecine, Marseille

3- Service des urgences, Hôpital Nord, Marseille

Correspondance : Vitton Véronique, Service d'hépato-gastro-entérologie, Hôpital Nord. Chemin des Bourrely. 13015 Marseille Cedex 20

(France). Téléphone : + 33 (0)4 91968737 - Télécopie : + 33 (0)4.919613 11. Mailto : veronique.vitton@mail.ap-hm.fr 
(internes ou médecins seniors) rend son interprétation plus ou moins aisée.

\section{Recueil des données}

Notre démarche de recueil des besoins de formation s'est appuyée sur deux outils : des entrevues individuelles et un questionnaire ouvert, dont les réponses ont été hiérarchisées à l'aide d'une grille publiée, la grille FGP (Fréquence, gravité, problème) ${ }^{8}$.

\section{Entrevues individuelles}

Chaque médecin participant, senior ou interne, a accepté une entrevue individuelle dont la durée était fixée à dix minutes. L'entretien visait à recueillir la perception des professionnels à l'égard : 1) des difficultés rencontrées dans la prise en charge des urgences digestives au cours de leur pratique quotidienne ; 2) des besoins de formation perçus dans le domaine de la gastroentérologie.

\section{Questionnaires et grille d'analyse}

Nous avons utilisé un questionnaire ouvert, dont les réponses ont été analysées et hiérarchisées en se référant à une grille publiée et proposée par d'Ivernois ${ }^{8}$, largement reconnue et utilisée dans le contexte de la FMC en France, la grille FGP (fréquence, gravité, problèmes). Le recueil a débuté par une réunion qui avait deux objectifs. Le premier était de présenter le projet d'évaluation des besoins de formations et d'expliquer les finalités du questionnaire et de la grille à l'ensemble des participants. Le deuxième était de lister les sujets pouvant faire l'objet d'un programme d'enseignement. Un questionnaire ouvert, intégrant la grille, a ainsi été distribué aux participants. Dans la deuxième étape, chaque participant devait, pour chaque sujet retenu, coter selon son expérience personnelle : 1) la fréquence $(\mathrm{F}) ; 2$ ) la gravité $(G)$ et : 3) les problèmes $(P)$, concernant chaque sujet de la liste précédemment établie. Il devait ensuite additionner horizontalement sur le questionnaire les chiffres qu'il avait attribués aux trois colonnes FGP pour chaque sujet. Le tablean 1 représente le questionnaire FGP utilisé. Tous les questionnaires, recueillis à l'issue de cette réunion, étaient anonymes.

Nous avons utilisé le même questionnaire FGP pour recueillir le point de vue des gastroentérologues sur les problèmes qu'ils estimaient être rencontrés par les médecins urgentistes. La réunion préliminaire à l'administration des questionnaires au sein du service de gastroentérologie avait permis d'expliquer d'une part, le fonctionnement de la grille de réponse et, d'autre part, qu'il s'agissait d'estimer uniquement les problèmes rencontrés par les médecins urgentistes et non ceux rencontrés par les gastroentérologues eux-mêmes. L'ensemble des questionnaires a également été recueilli à l'issue de la réunion.

\section{Analyse des données}

Les données ont été saisies sur Excel et analysées par le logiciel SPPS 10.1. Les fréquences ont été calculées pour les variables qualitatives et les moyennes pour les variables quantitatives. Les réponses des médecins ont été analysées en fonction de leur statut et de leur spécialité. Les tests statistiques utilisés sont le test exact de Fisher et celui de Wilcoxon.

\section{Résultats}

Nous avons pu recueillir l'avis de 22 participants urgentistes (12 médecins seniors et 10 internes) et des six gastroentérologues du service (deux professeurs des universités-praticiens hospitaliers, deux praticiens hospitaliers, deux chefs de clinique des universités-assistants des hôpitaux).

\section{Réponses des urgentistes}

Les entrevues individuelles ont permis de mettre en évidence des besoins de formation ressentis par l'ensemble des médecins interrogés.

Concernant les questionnaires, à l'issue de notre réunion initiale (tour de table) avec les médecins urgentistes, trois sujets principaux ont été relevés : hémorragie digestive, pancréatite aiguë et angiocholite aiguë.

Les résultats sont détaillés dans le tableau 2.

Les médecins urgentistes exprimaient des besoins en termes de savoir (connaissances) et de savoir-faire (habiletés). Pour l'ensemble des urgentistes, aucun besoin dans le registre du savoir être (attitudes) n'a été relevé. Pour la majorité des items, les réponses des seniors et des internes étaient proches, sauf pour le champ du savoir faire concernant la pancréatite aiguë et l'angiocholite. En effet, pour ces deux sujets, les internes exprimaient des besoins plus importants de manière significative (respectivement : $\mathrm{p}<0.002$ et $\mathrm{p}<0.03$ ).

\section{Réponses des gastroentérologues}

Les gastroentérologues ont décrit, pour l'ensemble des items, des problèmes relatifs au savoir-être pour les urgentistes. Des problèmes de savoir et de savoirfaire sont également rapportés, et sont proches 


\section{Communication brève}

des réponses des urgentistes, sauf pour l'angiocholite pour laquelle les problèmes de savoir-faire sont cotés de manière plus importante que par les urgentistes eux- mêmes, avec une différence statistiquement significative $(\mathrm{p}<0.05)$.

Les résultats détaillés sont présentés dans le tableau 3.

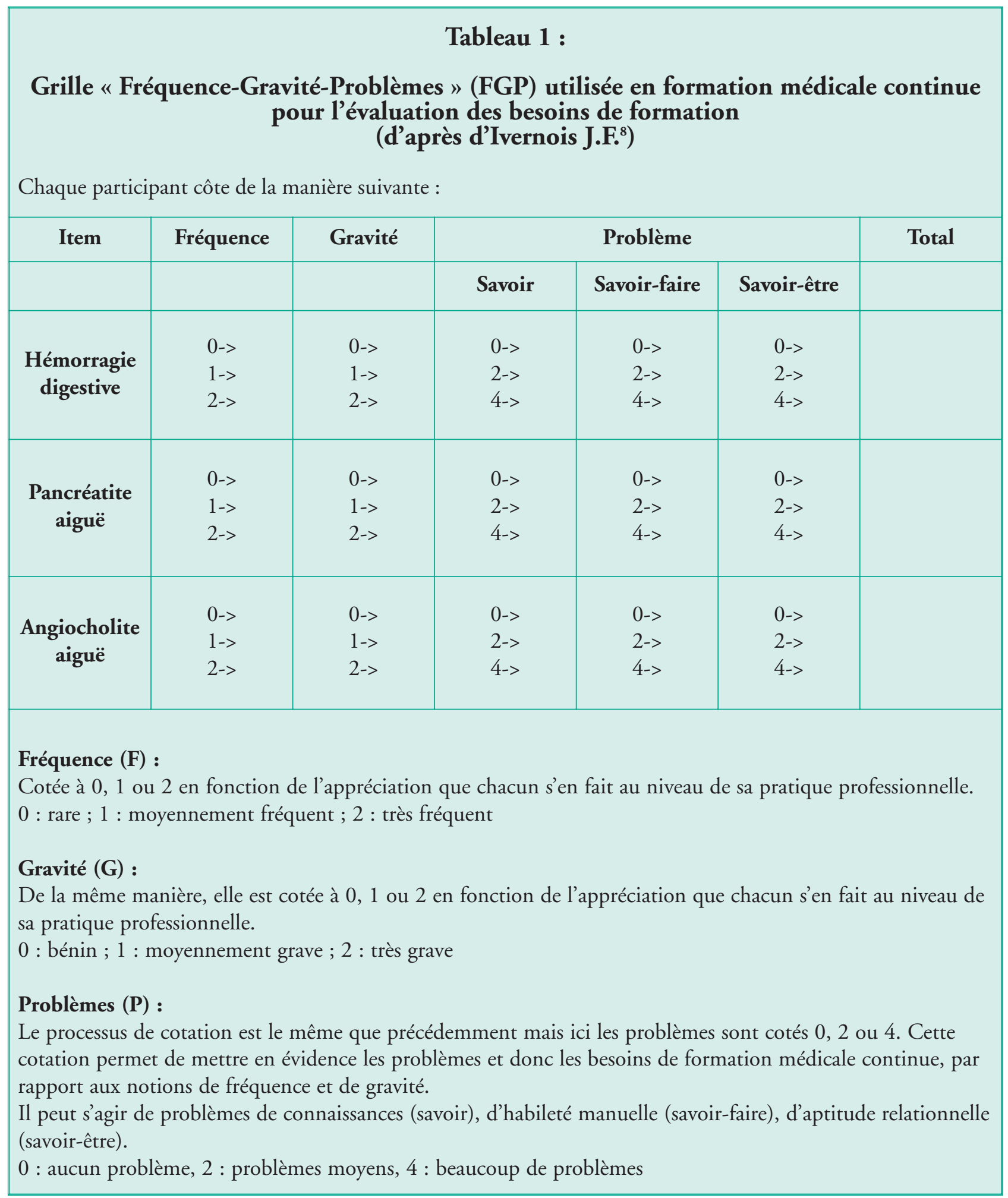


Evaluation préliminaire des besoins de formation en gastroentérologie...

Tableau 2 :

Moyennes des scores obtenus par items pour les seniors et les internes des urgences

\begin{tabular}{|c|c|c|c|c|}
\hline Items & & Séniors & Internes & Significativité \\
\hline \multirow{5}{*}{$\begin{array}{l}\text { Hémorragie } \\
\text { digestive }\end{array}$} & Fréquence & 1,92 & 2 & NS \\
\hline & Gravité & 1,67 & 1,8 & NS \\
\hline & Problème savoir & 3,67 & 3,6 & NS \\
\hline & $\begin{array}{l}\text { Problème } \\
\text { savoir-faire }\end{array}$ & 2,67 & 2,4 & NS \\
\hline & Problème savoir-être & 0 & 0 & NS \\
\hline \multirow{5}{*}{ Pancréatite aigüe } & Fréquence & 1,58 & 1,5 & NS \\
\hline & Gravité & 2 & 2 & NS \\
\hline & Problème savoir & 3,83 & 4 & NS \\
\hline & $\begin{array}{l}\text { Problème } \\
\text { savoir-faire }\end{array}$ & 2 & 3,2 & 0,002 \\
\hline & Problème savoir-être & 0 & 0 & NS \\
\hline \multirow{5}{*}{ Angiocholite aiguë } & Fréquence & 1,58 & 1,5 & NS \\
\hline & Gravité & 2 & 2 & NS \\
\hline & Problème savoir & 3,33 & 3,6 & NS \\
\hline & $\begin{array}{l}\text { Problème } \\
\text { savoir-faire }\end{array}$ & 3,17 & 4 & 0,023 \\
\hline & Problème savoir-être & 0 & 0 & NS \\
\hline 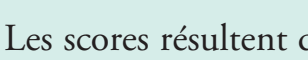 & $1 . \quad . \quad 1$ & & & \\
\hline
\end{tabular}




\section{Communication brève}

Tableau 3 :

Moyennes des scores obtenus par items "problèmes » par les urgentistes (seniors+internes) et les gastroentérologues

\begin{tabular}{|c|c|c|c|c|}
\hline Items & & Urgentistes & Gastroentérologues & Significativité \\
\hline \multirow{3}{*}{$\begin{array}{l}\text { Hémorragie } \\
\text { digestive }\end{array}$} & Problème savoir & 3,64 & 3 & NS \\
\hline & $\begin{array}{l}\text { Problème } \\
\text { savoir-faire }\end{array}$ & 2,55 & 3,33 & NS \\
\hline & $\begin{array}{l}\text { Problème } \\
\text { savoir-être }\end{array}$ & 0 & 1 & 0,001 \\
\hline \multirow{3}{*}{ Pancréatite aigüe } & Problème savoir & 3,91 & 2,33 & 0 \\
\hline & $\begin{array}{l}\text { Problème } \\
\text { savoir-faire }\end{array}$ & 2,55 & 2,67 & NS \\
\hline & $\begin{array}{l}\text { Problème } \\
\text { savoir-être }\end{array}$ & 0 & 1 & 0,001 \\
\hline \multirow{3}{*}{ Angiocholite aiguë } & Problème savoir & 3,45 & 2,67 & NS \\
\hline & $\begin{array}{l}\text { Problème } \\
\text { savoir-faire }\end{array}$ & 3,55 & 2 & 0,001 \\
\hline & $\begin{array}{l}\text { Problème } \\
\text { savoir-être }\end{array}$ & 0 & 1,33 & 0,001 \\
\hline
\end{tabular}

Les scores résultent d'une application de la grille $\mathrm{FGP}^{8}$ 


\section{Evaluation préliminaire des besoins de formation en gastroentérologie...}

\section{Discussion}

Notre travail a permis de démontrer l'existence d'un besoin de formation en gastro-entérologie des médecins urgentistes. Les résultats obtenus ont montré que les médecins urgentistes exprimaient à la fois des besoins en termes de connaissances (savoir) et d'habiletés (savoirfaire). Ces besoins étaient présents quel que soit le sujet considéré. Pour deux des sujets proposés, pancréatite aiguë et angiocholite, les besoins des internes étaient supérieurs à ceux des médecins seniors de manière significative. En revanche, aucun besoin en termes de savoir-être, c'est-àdire d'aptitude relationnelle, n'a été exprimé. Ces résultats étaient donc en faveur d'une carence de connaissances théoriques, en lien avec une absence d'attitude claire vis-àvis de la prise en charge des urgences digestives. Le point de vue des gastroentérologues du service était assez différent, dans la mesure où ces derniers ont estimé que les urgentistes rencontraient également des problèmes dans le registre du savoir être pour les trois sujets proposés. Cette constatation était un peu surprenante et semblait surtout à rapprocher d'un manque d'information donnée par les urgentistes aux patients sur la suite de leur prise en charge en service spécialisé. Néanmoins, il est possible que ce déficit perçu par les gastroentérologues concernant leurs confrères urgentistes s'explique par un manque de connaissances théoriques spécifiques, empêchant une information claire des patients. Les gastroentérologues décrivaient également des problèmes de savoir et de savoir faire de la part des urgentistes dans une proportion identique, sauf pour l'angiocholite pour laquelle ils décrivaient des problèmes de savoir faire significativement supérieurs à ceux estimés par les urgentistes eux-mêmes. Cette constatation pourrait nous permettre d'insister de manière plus importante sur ce sujet lors de l'élaboration du projet de formation.

Notre démarche d'évaluation des besoins de formation avait débuté après la constatation répétée d'une inadéquation entre la prise en charge des urgences digestives par les médecins urgentistes et celle préconisée par notre service. Les causes de cette discordance restaient cependant à analyser.

Plusieurs outils sont disponibles pour évaluer un besoin de formation : questionnaires, test de connaissances, entrevues individuelles, évaluation des pratiques professionnelles, mise en situation, technique d'incidents critiques, discussions de groupes ${ }^{9-11}$. Nous avions choisi d'utiliser, dans ce travail préliminaire, deux outils d'évaluation : les entretiens individuels et un questionnaire. Dans un premier temps nous avions pu interroger l'ensemble des médecins urgentistes (seniors et internes) en entretiens individuels courts. Ces derniers ont permis de mettre en évidence des besoins ressentis de formation de la part de tous les médecins interrogés. Ces entrevues ont en effet permis de confirmer que l'ensemble des médecins urgentistes estimait rencontrer régulièrement des difficultés dans la prise en charge des urgences digestives. De plus, tous étaient favorables à la mise en place d'un projet de formation. Dans un second temps nous avons utilisé la technique du questionnaire. C'est une méthode qui a pour avantage d'être simple, facile à exploiter et anonyme. Elle nous a paru également bien adaptée à la population étudiée en raison du manque de disponibilité des médecins participants. Ses limites sont d'être une technique fermée et non interactive, ce qui peut limiter la discussion. Nous avons choisi d'utiliser la grille " fréquence, gravité, problème " (FGP), largement reconnue et employée en France pour l'évaluation des besoins de formation en formation médicale continue ${ }^{9}$. L'utilisation de cette grille, précédée d'une réunion avec les participants et complétée par les entretiens individuels préalables peut toutefois en partie limiter cet écueil. Elle a également permis de mettre en évidence des besoins exprimés par la majorité des médecins interrogés. L'utilisation d'une grille identique par les gastroentérologues est discutable puisqu'il ne s'agit pas d'une évaluation de leurs besoins. Néanmoins il nous a paru intéressant d'utiliser le même support pour comparer les points de vue des apprenants et des enseignants.

Notre travail présente quelques limites. En effet, l'échantillon de médecins interrogés était faible et notre étude n'a porté que sur un seul service d'urgences. Par ailleurs, nous n'avons utilisé que deux outils d'évaluation des besoins de formation alors que la multiplicité de ces outils est fondamentale pour évaluer plus précisément les besoins de formation exprimés et ressentis. Cependant, il s'agissait d'un travail d'évaluation préliminaire qui nous a permis d'identifier des pistes pour le développement d'une formation des médecins urgentistes en gastroentérologie. Ultérieurement, l'utilisation d'autres outils d'évaluation des besoins de formation, en particulier la technique d'incidents critiques devrait nous permettre de mieux identifier les problèmes réels auxquels les médecins urgentistes sont confrontés au cours de leur pratique quotidienne et d'ébaucher une évaluation des pratiques professionnelles.

La perspective de ce travail sera de planifier une formation pertinente des médecins urgentistes. Elle nécessitera une définition rigoureuse des objectifs d'enseignements qui ne peuvent se concevoir qu'après l'évaluation des 


\section{Communication brève}

besoins ressentis et exprimés. Cette formation pourra être étendue à l'ensemble des services d'urgences des $\mathrm{CHU}$ marseillais. Dans un avenir plus lointain, si cette formation rencontre le succès escompté, nous pourrons également envisager de l'étendre aux autres services de spécialités médicales et chirurgicales.

\section{Remerciements}

Nous remercions le Pr Jean-Charles Grimaud, les Drs Philippe Jean et Philippe Cano pour leur soutien à ce projet ainsi que pour leurs conseils pour la réalisation de ce travail.

\section{Contributions}

Véronique Vitton a conçu le protocole d'évaluation, recueilli les données, interprété les résultats et rédigé le manuscrit. Pascal Vignally et Stéphanie Gentile ont participé à l'interprétation des résultats et à l'analyse statistique. Maéva Delaveau et Jérôme Poussard ont contribué à la mise à la mise en place des réunions de travail et au recueil des données. Marc Barthet a participé à la conception du protocole et à la révision du manuscrit.

\section{Références}

1. Code de déontologie médicale. In: Code de la santé publique (numéros R.4127-1 à R.4127-112). Mise à jour du 14 déc. 2006). [On-line]. Disponible sur: http://www.web.ordre.medecin.fr/deonto/decret/codedeont.pdf

2. Loi $n^{\circ}$ 2002-303 du 4 mars 2002 relative aux droits des malades et à la qualité du système de santé (NoR: MESX0100092L). Journal Officiel de la République Française, 5 mars 2002. [On-line]. Disponible sur: http://www.legifrance.gouv.fritexteconsolide/SPECF.htm

3. Jean P. Pour une planification méthodique des activités de formation. Pédagogie Médicale 2001;2:101-7.

4. Norman GR, Shannon SI, Marrin ML. The need for needs assesment in continuing medical education. BMJ 2004;328:999-1001.

5. Jouquan J. La problématique de l'analyse des besoins de formation. Pédagogie Médicale 2004;5:133-5.

6. Ministère de l'éducation. Etudes médicales. Objectifs pédagogiques terminaux pour les items de la deuxième partie du deuxième cycle des études médicales. B.O.n ${ }^{\circ}$ 31-30/08/2001.
7. Gerbeaux P, Zanini D, Torro D, Rakotonirina J, Nehl P, Gambini $G$ et al. Méthodologie d'élaboration d'un programme pédagogique pour l'apprentissage hospitalier de la médecine d'urgence. Pédagogie Médicale 2003;4:97-102.

8. d'Ivernois JF. Un instrument pour l'évaluation des besoins de formation en matière de FMC. Bulletin de l'ASFORMED $1978 ; n^{\circ}$ 8. In: Concours Médicale $1978 ; 100: 7383-87.6$.

9. Honnorat C, Levasseur G. Quels besoins enseigner, quel besoin d'enseigner ? Pédagogie Médicale 2001;2:26-30.

10. Shahabudin SH. Designing in continuing medical education programme in Malaysia based on practitioners' needs. Med Educ 1990;24:264-70.

11. Dunn WR, Hamilton DD. The Critical Incident Technique-a brief guide. Med Teach 1986;8:207-15.

Manuscrit reçu le 28 avril 2005 ; commentaires éditoriaux formulés aux auteurs le 29 septembre 2005 ; accepté pour publication le 10 mars 2007. 